\title{
Traduire
}

Une autre perspective sur r tr traduction

Revue française de la traduction

$221 \mid 2009$

Voies de l'interprétation

\section{Rétrospective $\mathrm{n}^{\circ} 15$}

\section{Maurice Voituriez}

\section{(2) OpenEdition \\ Journals}

Édition électronique

URL : http://journals.openedition.org/traduire/363

DOI : 10.4000/traduire.363

ISSN : 2272-9992

\section{Éditeur}

Société française des traducteurs

\section{Édition imprimée}

Date de publication : 15 décembre 2009

Pagination : 120-122

ISSN : 0395-773X

\section{Référence électronique}

Maurice Voituriez, «Rétrospective n 15 », Traduire [En ligne], 221 | 2009, mis en ligne le 12 novembre 2013, consulté le 02 décembre 2020. URL : http://journals.openedition.org/traduire/363 ; DOI : https:// doi.org/10.4000/traduire.363 


\section{Rétrospective $n^{\circ} 15$}

\section{Maurice Voituriez}

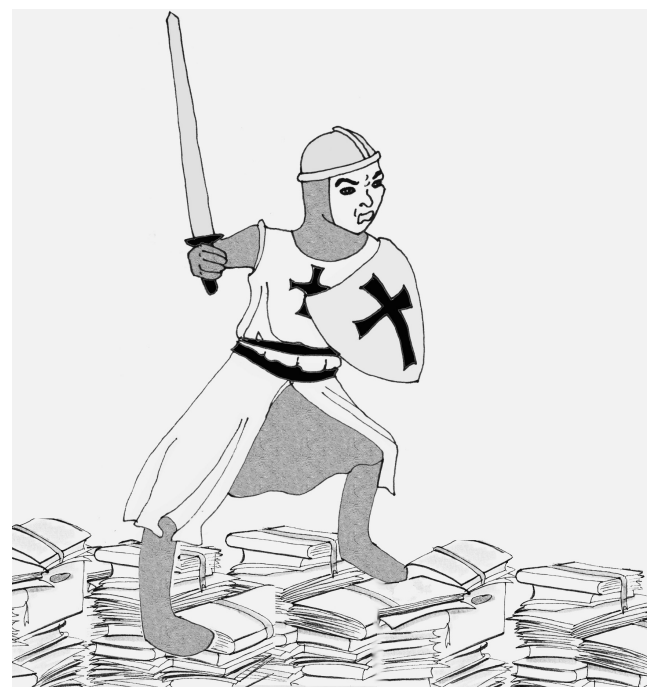

Illustration : Marlène Junius, http://alotoftralala.over-blog.com

" La traduction est plus qu'une profession, c'est une véritable industrie qui, en fait de titres de noblesse, a ses chevaliers".

Cette citation, assez malicieuse, ouvrant le No 135 de notre revue, émane de John P. Oatmill. Ce nom ne vous dit rien ? Cela n'est pas étonnant. Nous le connaissons car il s'agit de l'anagramme de notre ami, le commandant Jean Maillot, toujours chez nous à l'époque, directeur de la publication. Déjà le terme de " chevalier d'industrie " laissait subodorer une culture française. Non qu'il n'en existe pas d'équivalent ailleurs. II y a en allemand le Hochstapler (le Felix Krull, de Thomas Mann). Toute profession a ses imposteurs mais heureusement ils constituent de négligeables exceptions. 
En revanche, tous les traducteurs sont mortels, ce qui fait que presque chaque numéro de notre revue contient des hommages aux disparus. Maurice Gravier et Florence Herbulot retracent la carrière de Daniel Moskowitz, traducteur technique, directeur de l'ESIT pour la traduction.

La SFT accueille aussi en son sein les traducteurs littéraires. Son fondateur en était un. Marguerite Yourcenar était également membre d'honneur de notre syndicat. C'est à ce titre que Traduire lui rend hommage in memoriam.

On dit parfois que peu de traducteurs littéraires jouissent, comme tels, d'une grande notoriété. Souvent on ignore même qu'un écrivain célèbre a traduit. Nous pensons à R.M. Rilke et à André Gide. Dans le cas de Marguerite Yourcenar la situation est un peu différente. Certes elle restera pour beaucoup l'auteur des Mémoires d'Hadrien et de L'OEuvre au Noir mais on sait généralement que (suivant les traces de madame Dacier, la première femme traductrice connue, celle qui au XVIII siècle fit connaître Homère aux Français), dans La Couronne et la Lyre elle nous présenta les poètes grecs anciens. Elle avait aussi traduit Les Vagues de Virginia Woolf.

Née en Belgique, d'un père français et d'une mère belge, elle avait vécu en Flandre française, dans cette belle région du Mont-Noir (car notre Flandre maritime est aussi pays de collines !) et près du village de Saint-Jans-Cappel, où un musée lui était déjà consacré depuis quelques temps. Cette académicienne (la première en France à appartenir aux Quarante Immortels !) fréquentait davantage la Flandre que le Quai Conti !

Revenant sur la personnalité de P.-F. Caillé, notre fondateur, nous mentionnons l'étude consacrée par Mlle Imma Kunz à l'activité de ce dernier dans les domaines du doublage et du soustitrage et de l'adaptation de films. Plus de trois cents, dont: Autant en emporte le vent (évidemment !), West Side Story, Guerre et Paix, Un Roi à New York, Zorro, etc. La liste se serait encore allongée si la télévision n'en était pas encore en France au stade de l'enfance! Dans son Index Translationum, I'UNESCO répertorie périodiquement les ouvrages traduits.

Cela n'étonnera personne : c'est la Bible qui restait en 1985 l'ouvrage le plus traduit, avec 244 versions. Les ouvrages religieux avaient alors une audience internationale et, parmi les contemporains français, Teilhard de Chardin et Jacques Maritain ont été, l'un et l'autre, traduits en sept langues.

Après la religion vient la politique. Ce ne sont ni Karl Marx, ni Mao Tse-Tung qui ont la vedette mais Lénine... avec 406 versions. Attention ! II s'agit du nombre de langues mais la diffusion constitue un autre aspect du problème et la Bible - du seul fait de son ancienneté - a connu une diffusion mondiale bien plus considérable que l'oeuvre de Lénine. Ajoutons-y le fait que la Vulgate (donc la traduction latine établie par notre patron saint Jérôme) a longtemps été la référence de l'Eglise.

Après la religion et la politique, c'est la science-fiction qui sucite le plus d'intérêt dans le monde entier. Quatre-vingts ans après sa mort, Jules Verne reste un des auteurs les plus traduits au monde, avec 229 traductions. 
N'oublions pas non plus que le fait pour un auteur d'entrer dans le domaine public donne à son œuvre un " second souffle " pour d'évidentes raisons commerciales. C'est ainsi qu'au xxe siècle les Contes de Grimm connaissent un regain de succès.

D'une façon générale les écrivains pour la jeunesse furent longtemps les plus traduits et les moins démodés mais le succès de la télévision auprès des jeunes a dû modifier la situation depuis 1988 (année où parut l'article de Traduire à ce sujet). Pourtant certaines évolutions se dessinaient ; c'est le cas de le dire si l'on parle de la B.D. : Walt Disney avait alors été traduit 137 fois et Goscinny 89 fois.

C'est le roman policier qui, depuis le xxe siècle, a suscité la majeure partie des traductions. Agatha Christie et Simenon occupaient déjà une place d'honneur.

Quant à la littérature " classique ", elle se porte toujours bien. Tolstoï vient en tête, suivi par Shakespeare. Molière est moins traduit qu'Alexandre Dumas mais Victor Hugo reste en bonne place. Parmi les Français contemporains Proust et Sartre semblent également appréciés.

II ne faut pas perdre ceci de vue : le fait que des œuvres soient traduites ne prouve rien en faveur du rayonnement d'une langue. Au contraire ! Le jour où, par hypothèse, tous les Européens connaitraient l'anglais, cette langue n'aurait plus besoin de traducteurs sur notre continent. Le nombre de traductions d'une œuvre prouve uniquement sa valeur ou simplement l'intérêt qu'elle suscite chez des éditeurs avisés. Combien d'Occidentaux savent-ils lire Jaroslav Hasek, (l'auteur du Bon soldat Schweik) ou Knut Hamsun ou Félix Timmermans dans le texte original ? Inversement, dans des pays comme les Pays-Bas beaucoup de gens cultivés sont à même de lire les romans français, anglais ou allemands dans le texte, ce qui y limite le marché de la traduction... et de l'édition nationale.

Sous le titre "Les dix règles du langage informe(atique) ", Marc Boyer dénonce le sabir qui sévissait déjà en ce domaine. Hélas, en vingt ans la situation s'est aggravée. Notre opinionelle vaut ce qu'elle vaut ! - est de faire confiance à l'utilisateur francophone qui, peu doué pour les langues, en général, moins encore pour l'américain technique ou pseudo-technique, en particulier, inventera un langage, le plus souvent un jargon, mais " bien de chez nous".

La diffusion explosive et irrésistible de cette informatique dans les pays francophones encourage peu à peu le recours à des termes parfois peu académiques mais faciles à prononcer et à entendre. Plus d'une technique a connu cette évolution ! 